LPTHE/99-41

\title{
Correlators in integrable quantum field theory. The scaling RSOS models
}

\author{
G. Delfino \\ Laboratoire de Physique Théorique et Hautes Energies \\ Université Pierre et Marie Curie, Tour $161^{\text {er }}$ étage, 4 place Jussieu \\ 75252 Paris cedex 05, France \\ E-mail: aldo@lpthe.jussieu.fr
}

\begin{abstract}
The study of the scaling limit of two-dimensional models of statistical mechanics within the framework of integrable field theory is illustrated through the example of the RSOS models. Starting from the exact description of regime III in terms of colliding particles, we compute the correlation functions of the thermal, $\varphi_{1,2}$ and (for some cases) spin operators in the two-particle approximation. The accuracy obtained for the moments of these correlators is analysed by computing the central charge and the scaling dimensions and comparing with the exact results. We further consider the (generally non-integrable) perturbation of the critical points with both the operators $\varphi_{1,3}$ and $\varphi_{1,2}$ and locate the branches solved on the lattice within the associated two-dimensional phase diagram. Finally we discuss the fact that the RSOS models, the dilute $q$-state Potts model at and the $O(n)$ vector model are all described by the same perturbed conformal field theory.
\end{abstract}




\section{Introduction}

Integrable field theory emerged in the last years as an elegant and effective tool for the study of many two-dimensional statistical models directly in their scaling limit. The approach relies on the fact that a large class of quantum field theories in $(1+1)$ dimensions admits an infinite number of integrals of motions (i.e. they are 'integrable') and can be completely solved on-shell [ [, 2]. The matrix elements of local operators on the asymptotic states are also exactly computable [3, 4] and lead to spectral series for the correlation functions whose quantitative effectiveness is remarkable. Among the statistical models that have been studied in this framework we mention the Ising model in a magnetic field [2, 5, 6, 7, 8], the $q$-state Potts model [9, 10, 11, 12], the $O(n)$ model [13, 14], and the Ashkin-Teller model [15].

It is the purpose of this paper to illustrate how the programme outlined above applies to the "restricted solid-on-solid" (RSOS) models introduced by Andrews, Baxter and Forrester in Ref. [16]. For any integer $p \geq 3$ they are defined on the square lattice in terms of a spin or "height" variable $h_{i}$ located at each site $i$ and taking the integer values from 1 to $p$. The heights of two nearest-neighbour sites $i$ and $j$ are constrained by the condition

$$
\left|h_{i}-h_{j}\right|=1
$$

which, in particular, leads to a natural splitting of the lattice into two sublattices on which the heights are all even or all odd. The Hamiltonian is further specified by a one-site and a diagonal interaction terms. Their precise form does need to be reproduced here but it is important that the energy of a configuration is invariant under the global tranformation

$$
h_{i} \rightarrow p+1-h_{i}
$$

which is the basic symmetry of the models.

In their general formulation the RSOS models contain a number of parameters which grows linearly with $p$. In Ref. [16] the models were solved on two two-dimensional manifolds of the parameter space which can be parameterised by a temperature-like variable $t$ together with a second coordinate $v$ measuring the spatial anisotropy of the lattice interaction. The scaling limit, however, is isotropic and $v$ will be ignored in the following. Hence, for each of the two solutions and for any $p$, the phase diagram reduces to a line and exhibits a critical point separating two phases known as regimes I and II (for the first solution) and regimes III and IV (for the second solution). Here, we will only be interested in the second case, and more specifically in regime III. 
By comparison of critical exponents, Huse showed [17] that the critical points separating regimes III and IV for the different values of $p$ correspond to the minimal unitary series of conformal field theories characterised by the values of the central charge 18, 19]

$$
C=1-\frac{6}{p(p+1)}, \quad p=3,4, \ldots .
$$

These models contain a finite number of primary operators $\varphi_{m, n}(x)(m=1, \ldots, p-1$; $n=1, \ldots, p)$ with scaling dimensions

$$
X_{m, n}=\frac{[(p+1) m-p n]^{2}-1}{2 p(p+1)} .
$$

It was shown in Ref. [20] that the unitary minimal models admit the Landau-Ginzburg description

$$
S=\int d^{2} x\left[(\partial \varphi)^{2}+\sum_{k=1}^{p-1} g_{k} \varphi^{2 k}\right],
$$

with $g_{1}=g_{2}=\cdots=g_{p-2}=0$. The scalar field $\varphi(x)$ is then the continous version of the shifted height variable $h_{i}-(p+1) / 2$, in such a way that the reflection symmetry (11.2) is mapped into $\varphi \rightarrow-\varphi$. The following identifications between normal ordered powers of $\varphi$ and conformal operators hold 20]

$$
\varphi^{k} \sim \varphi_{k+1, k+1}, \quad k=0, \ldots, p-2 .
$$

The effective action (1.5) makes transparent that the considered series of critical points corresponds to the $(p-1)$-critical behaviour of a system with scalar order parameter and $Z_{2}$ symmetry, $p=3$ being the ordinary Ising universality class, $p=4$ the tricritical Ising, and so on.

The RSOS models in regimes III and IV possess $p-1$ and $p-2$ degenerate ground states, respectively [16], and can be described by the action (1.5) with suitable fine tunings of the couplings $g_{k}$ leading to the appropriate number of degenerate minima in the effective potential. For $p=3$ the two regimes correspond to the low- and high-temperature phases of the Ising model in zero magnetic field and are both massive in the scaling limit. For $p \geq 4$, while regime III is still massive, regime IV become massless and corresponds to the crossover between the critical points labelled by $p$ and $p-1$ [21, 22].

Solvability on the lattice naturally suggests integrability of the field theory describing the scaling limit. In fact, the scaling dimension of the 'thermal' operator (conjugated to $t$ ) is known from the lattice solution and coincide with $X_{1,3}$, so that the scaling limit of the RSOS models in regimes III and IV is described by the action

$$
\mathcal{A}=\mathcal{A}_{C F T}^{(p)}+\lambda \int d^{2} x \varphi_{1,3}(x),
$$


where $\mathcal{A}_{C F T}^{(p)}$ is the action of the conformal theories with central charge (1.3), and $\lambda$ is a coupling with dimensions $m^{2-X_{1,3}}$. This $\varphi_{1,3}$ perturbation of conformal field theory is known to be massive (regime III) or massless (regime IV) depending on the sign of $\lambda$ [21], and to be integrable in both directions [2]. The associated scattering theories are also known [23, 24, 25].

The paper is organised as follows. In the next section we briefly review the exact scattering description for regime III and use it in Section 3 for the computation of form factors of the operators $\varphi_{1,3}, \varphi_{1,2}$ and (for $\left.p=3,4\right) \varphi_{2,2}$. In Section 4 we write down the two-particle approximation for the correlation functions of these operators and analyse its accuracy by computing the central charge and the scaling dimensions. Section 5 is devoted to a discussion of the perturbation of the RSOS critical points with both the operators $\varphi_{1,3}$ and $\varphi_{1,2}$. In the final section we briefly discuss the fact that the perturbed conformal field theory (10.7) also describe the scaling limit of the dilute $q$-state Potts model along its first order phase transition lines for $0 \leq q \leq 4$, as well as the $O(n)$ vector model for $-2 \leq n \leq 2$.

\section{Scattering theory}

In a $(1+1)$-dimensional theory with degenerate vacua the elementary excitations are kinks interpolating among these vacua. It is known from the lattice solution that the $j$-th ground state in regime III $(j=1, \ldots, p-1)$ is such that all the sites on one sublattice have height $j$ and all the sites on the other sublattice have height $j+1$ (Fig. 1). The space-time trajectory of a kink is a domain wall separating two different ground states. Since the pairing of two different ground states $i$ and $j$ can give an admissible configuration only if $|i-j|$ equals 1 (Fig. 2), the elementary excitations of the scattering theory are kinks $K_{i j}(\theta)$ interpolating between two vacua $i$ and $j=i \pm 1$. It follows from the precise form of the lattice interaction that the interfacial tension between two ground states $i$ and $i+1$ does not depend on $i$, and this amounts to say that the kinks $K_{i, i \pm 1}$ all have the same mass. Multikink excitations of the type

$$
\ldots K_{i \pm 1, i}\left(\theta_{1}\right) K_{i, i \pm 1}\left(\theta_{2}\right) \ldots
$$

will connect ground states with arbitrary indices.

\footnotetext{
${ }^{1}$ The rapidity variable $\theta$ parameterises the on-shell momenta of the kink of mass $m$ as $\left(p^{0}, p^{1}\right)=$ $(m \cosh \theta, m \sinh \theta)$.
} 
In an integrable field theory the scattering is completely elastic (no production processes allowed) and multiparticle processes factorise into the product of the two-body subprocesses, so that the problem of the determination of the $S$-matrix is reduced to the computation of the two-kink amplitudes [1]. Taking into account the kink composition rules together with invariance under time reversal and spatial inversion, the allowed twokink processes are those depicted in Fig. 3 and associated to the commutation relations

$$
\begin{aligned}
K_{j, j \pm 1}\left(\theta_{1}\right) K_{j \pm 1, j}\left(\theta_{2}\right) & =A_{j}^{ \pm}\left(\theta_{1}-\theta_{2}\right) K_{j, j \pm 1}\left(\theta_{2}\right) K_{j \pm 1, j}\left(\theta_{1}\right) \\
& +B_{j}\left(\theta_{1}-\theta_{2}\right) K_{j, j \mp 1}\left(\theta_{2}\right) K_{j \mp 1, j}\left(\theta_{1}\right), \\
K_{j \pm 1, j}\left(\theta_{1}\right) K_{j, j \mp 1}\left(\theta_{2}\right) & =C_{j}\left(\theta_{1}-\theta_{2}\right) K_{j \pm 1, j}\left(\theta_{2}\right) K_{j, j \mp 1}\left(\theta_{1}\right) .
\end{aligned}
$$

The scattering amplitudes are subject to a series of constraints. Invariance under the reflection $j \rightarrow p-j$ requires

$$
\begin{aligned}
& A_{j}^{+}(\theta)=A_{p-j}^{-}(\theta), \\
& B_{j}(\theta)=B_{p-j}(\theta), \\
& C_{j}(\theta)=C_{p-j}(\theta),
\end{aligned}
$$

while crossing symmetry implies

$$
\begin{aligned}
& A_{j}^{ \pm}(\theta)=A_{j \pm 1}^{\mp}(i \pi-\theta), \\
& B_{j}(\theta)=C_{j}(i \pi-\theta) .
\end{aligned}
$$

Commuting once again the r.h.s. of Eqs. (2.3) and (2.4) leads to the unitarity equations

$$
\begin{aligned}
A_{j}^{ \pm}(\theta) A_{j}^{ \pm}(-\theta)+B_{j}(\theta) B_{j}(-\theta) & =1, \\
A_{j}^{ \pm}(\theta) B_{j}(-\theta)+B_{j}(\theta) A_{j}^{\mp}(-\theta) & =0, \\
C_{j}(\theta) C_{j}(-\theta) & =1 .
\end{aligned}
$$

A three-kink process can be factorised in two ways differing by the ordering of the twobody collisions. Equating the results leads to the factorisation equation

$$
A_{j}^{ \pm} A_{j \pm 1}^{\mp} A_{j}^{ \pm}+B_{j} C_{j} B_{j}=A_{j \pm 1}^{\mp} A_{j}^{ \pm} A_{j \pm 1}^{\mp}+B_{j \pm 1} C_{j \pm 1} B_{j \pm 1},
$$

and similar others (the arguments of the three factors in each product are $\theta, \theta+\theta^{\prime}$ and $\theta^{\prime}$, respectively). 
The minimal solution to all these requirements is well known [16, 23, 24] and reads

$$
\begin{aligned}
& A_{j}^{ \pm}(\theta)=\left(\frac{s_{j \pm 1}}{s_{j}}\right)^{i \theta / \pi} \frac{s_{1}}{s_{j}} \frac{\sinh \frac{1}{p}(i j \pi \pm \theta)}{\sinh \frac{1}{p}(i \pi-\theta)} S_{0}(\theta), \\
& B_{j}(\theta)=\left(\frac{\sqrt{s_{j+1} s_{j-1}}}{s_{j}}\right)^{1+i \theta / \pi} \frac{\sinh \frac{\theta}{p}}{\sinh \frac{1}{p}(i \pi-\theta)} S_{0}(\theta), \\
& C_{j}(\theta)=\left(\frac{\sqrt{s_{j+1} s_{j-1}}}{s_{j}}\right)^{-i \theta / \pi} \frac{\sinh \frac{1}{p}(i \pi-\theta)}{\sinh \frac{1}{p}(i \pi-\theta)} S_{0}(\theta),
\end{aligned}
$$

where

$$
\begin{gathered}
s_{j} \equiv \sin \frac{j \pi}{p}, \\
S_{0}(\theta)=-\prod_{n=0}^{\infty} \frac{\Gamma\left(1+\frac{2}{p}\left(n+\frac{1}{2}\right)+\frac{\theta}{i \pi p}\right) \Gamma\left(1+\frac{2}{p} n-\frac{\theta}{i \pi p}\right)}{\Gamma\left(1+\frac{2}{p}\left(n+\frac{1}{2}\right)-\frac{\theta}{i \pi p}\right) \Gamma\left(1+\frac{2}{p} n+\frac{\theta}{i \pi p}\right)} \\
\times \frac{\Gamma\left(\frac{2}{p}(n+1)-\frac{\theta}{i \pi p}\right) \Gamma\left(\frac{2}{p}\left(n+\frac{1}{2}\right)+\frac{\theta}{i \pi p}\right)}{\Gamma\left(\frac{2}{p}(n+1)+\frac{\theta}{i \pi p}\right) \Gamma\left(\frac{2}{p}\left(n+\frac{1}{2}\right)-\frac{\theta}{i \pi p}\right)} \\
=-\exp \left\{i \int_{0}^{\infty} \frac{d x}{x} \frac{\sinh (p-1) \frac{x}{2}}{\sinh \frac{p x}{2} \cosh \frac{x}{2}} \sin \frac{x \theta}{\pi}\right\} .
\end{gathered}
$$

It can be checked that the amplitudes do not posses poles in the physical strip $\operatorname{Im} \theta \in(0, \pi)$, what ensures that there are no bound states and that the amplitudes given above entirely determine the scattering theory.

\section{Form factors}

Let us denote $\Phi(x)$ a local scalar operator of the theory with zero topological charge, namely such that its action on the vacuum $\left|0_{j}\right\rangle$ only produces excitations beginning and ending on this vacuum. All the operators we will consider in the following share this property. We are interested in the two-particle form factors (Fig. 4)

$$
F_{j, \pm}^{\Phi}\left(\theta_{1}-\theta_{2}\right)=\left\langle 0_{j}|\Phi(0)| K_{j, j \pm 1}\left(\theta_{1}\right) K_{j \pm 1, j}\left(\theta_{2}\right)\right\rangle .
$$

Eq. (2.3) implies the relation

$$
F_{j, \pm}^{\Phi}(\theta)=A_{j}^{ \pm}(\theta) F_{j, \pm}^{\Phi}(-\theta)+B_{j}(\theta) F_{j, \mp}^{\Phi}(-\theta),
$$

while crossing leads to the equations [10]

$$
F_{j, \pm}^{\Phi}(\theta+2 i \pi)=F_{j \pm 1, \mp}^{\Phi}(-\theta),
$$




$$
-i \operatorname{Res}_{\theta=i \pi} F_{j, \pm}^{\Phi}(\theta)=i \operatorname{Res}_{\theta=i \pi} F_{j \pm 1, \mp}^{\Phi}(\theta)=\left\langle 0_{j}|\Phi| 0_{j}\right\rangle-\left\langle 0_{j \pm 1}|\Phi| 0_{j \pm 1}\right\rangle .
$$

As a last necessary condition, the two-kink form factors are subject to the asymptotic bound $[5$

$$
\lim _{\theta \rightarrow+\infty} F_{j, \pm}^{\Phi}(\theta) \leq \text { constant } e^{X_{\Phi} \theta / 2},
$$

where $X_{\Phi}$ denotes the scaling dimension of the operator $\Phi(x)$.

It is easily checked that a class of solutions of Eqs. (3.2) and (3.3) is given by

$$
F_{j, \pm}^{\phi}=-\frac{2 i}{p}\left(\frac{s_{j \pm 1}}{s_{j}}\right)^{(1+i \theta / \pi) / 2} \frac{F_{0}(\theta)}{\sinh \frac{1}{p}(\theta-i \pi)} \Omega_{j}^{\phi}(\theta),
$$

where $\{\phi\} \subset\{\Phi\}$,

$$
F_{0}(\theta)=-i \sinh \frac{\theta}{2} \exp \left\{\int_{0}^{\infty} \frac{d x}{x} \frac{\sinh (1-p) \frac{x}{2}}{\sinh \frac{p x}{2} \cosh \frac{x}{2}} \frac{\sin ^{2}(i \pi-\theta) \frac{x}{2 \pi}}{\sinh x}\right\}
$$

is solution of the equations

$$
\begin{aligned}
& F_{0}(\theta)=S_{0}(\theta) F_{0}(-\theta), \\
& F_{0}(\theta+2 i \pi)=F_{0}(-\theta),
\end{aligned}
$$

and behave as

$$
F_{0}(\theta) \sim \exp [(1+1 / p) \theta / 4], \quad \theta \rightarrow+\infty .
$$

The functions $\Omega_{j}^{\phi}(\theta)$ are free of poles and satisfy

$$
\begin{aligned}
& \Omega_{j}^{\phi}(\theta)=\Omega_{j}^{\phi}(-\theta), \\
& \Omega_{j}^{\phi}(\theta+2 i \pi)=-\Omega_{j \pm 1}^{\phi}(-\theta) .
\end{aligned}
$$

These requirements, together with (3.5) imply that the $\Omega_{j}^{\phi}(\theta)$ are polynomials in $\cosh (\theta / 2)$. Let us consider those operators $\phi(x)$ which are relevant in the renormalisation group sense $\left(X_{\phi}<2\right)$ for all values of $p \geq 3$. Then, the bound (3.5) implies that these polynomials are at most of degree one, which means that the operator subspace $\{\phi\}$ contains only two independent relevant operators. The trace of the stress-energy tensor $\Theta(x)$ is a relevant operator and is even under the reflection symmetry, so that $F_{j,+}^{\Theta}(\theta)=F_{p-j,-}^{\Theta}(\theta)$. Moreover $\left\langle 0_{j}|\Theta| 0_{j}\right\rangle$ does not depend on $j$ and, according to (3.4), the two-kink matrix elements have no pole in $\theta=i \pi$. All these requirements are fulfilled if we take

$$
\Omega_{j}^{\Theta}(\theta)=2 \pi m^{2} \cosh \frac{\theta}{2},
$$


with the normalisation constant fixed by

$$
F_{j, \pm}^{\Theta}(i \pi)=2 \pi m^{2}
$$

The other independent relevant operator in $\{\phi\}$ (let us denote it $\mathcal{E}(x)$ ) corresponds to the constant solution

$$
\Omega_{j}^{\mathcal{E}}(\theta)=(-1)^{j}\left\langle 0_{1}|\mathcal{E}| 0_{1}\right\rangle
$$

The order parameter $\varphi(x)$ is the most relevant operator which changes sign under reflection, and this means in particular

$$
\begin{gathered}
\left\langle 0_{j}|\varphi| 0_{j}\right\rangle=-\left\langle 0_{p-j}|\varphi| 0_{p-j}\right\rangle, \\
F_{j,+}^{\varphi}(\theta)=-F_{p-j,-}^{\varphi}(\theta) .
\end{gathered}
$$

For generic values of $p$, these properties are incompatible with those of the space of solutions spanned by (3.6), (3.13), (3.15) and we conclude that $\varphi \notin\{\phi\}$. We do not dispose of the solution for $F_{j,+}^{\varphi}(\theta)$ for generic $p$ and just quote the result for the two simplest cases

$$
\begin{gathered}
F_{1,+}^{\varphi}(\theta)=i\left\langle 0_{1}|\varphi| 0_{1}\right\rangle \tanh \frac{\theta}{2}, \quad p=3 \\
F_{j,+}^{\varphi}(\theta)=\frac{(-1)^{j}}{2 \Upsilon_{j}(i \pi)}\left\langle 0_{1}|\varphi| 0_{1}\right\rangle\left(\frac{s_{j+1}}{s_{j}}\right)^{(1+i \theta / \pi) / 2} \frac{F_{0}(\theta)}{\cosh \frac{\theta}{2}} \Upsilon_{j}(\theta), \quad p=4 .
\end{gathered}
$$

The functions

$$
\Upsilon_{j}(\theta)=\exp \left\{\int_{0}^{\infty} \frac{d x}{x} \frac{\sin ^{2}[2 i \pi|2-j|-\theta] \frac{x}{2 \pi}}{\cosh x \sinh 2 x}\right\}
$$

satisfy the equations

$$
\begin{aligned}
& \Upsilon_{1}(\theta)=\Upsilon_{3}(\theta)=\frac{\sinh \frac{1}{4}(i \pi+\theta)}{\sinh \frac{1}{4}(i \pi-\theta)} \Upsilon_{1}(-\theta), \\
& \Upsilon_{1}(\theta+2 i \pi)=\Upsilon_{2}(\theta),
\end{aligned}
$$

and behave as $\exp (\theta / 8)$ when $\theta \rightarrow+\infty$. It can be checked that $F_{j, \pm}^{\varphi}(\theta)=F_{j, \pm}^{\mathcal{E}}(\theta)$ for $p=3$.

\section{Correlation functions}

The correlation functions are obtained using the resolution of the identity

$$
1=\sum_{n=0}^{\infty} \int_{\theta_{1}>\ldots>\theta_{n}} \frac{d \theta_{1} \ldots d \theta_{n}}{(2 \pi)^{n}}|n\rangle\langle n|
$$


to sum over all intermediate $n$-kink states $|n\rangle$. A two-point function reads

$$
\begin{aligned}
\left\langle 0_{j}\left|\Phi_{1}(x) \Phi_{2}(0)\right| 0_{j}\right\rangle & =\sum_{\varepsilon= \pm} \int_{\theta_{1}>\theta_{2}} \frac{d \theta_{1}}{2 \pi} \frac{d \theta_{2}}{2 \pi} F_{j, \varepsilon}^{\Phi_{1}}\left(\theta_{1}-\theta_{2}\right) F_{j, \varepsilon}^{\Phi_{2}}\left(\theta_{2}-\theta_{1}\right) e^{-|x| E_{2}} \\
& +O\left(e^{-4 m|x|}\right), \quad m|x| \gg 1
\end{aligned}
$$

where $E_{2}=m\left(\cosh \theta_{1}+\cosh \theta_{2}\right)$ is the energy of the two-kink asymptotic state. The "two-kink approximation" (4.3) is known to provide results of remarkable accuracy for integrated correlators (see [10] and references therein), as can be checked through the use of the sum rules [26, 27]

$$
\begin{aligned}
& C=\frac{3}{4 \pi} \int d^{2} x|x|^{2}\left\langle 0_{j}|\Theta(x) \Theta(0)| 0_{j}\right\rangle, \\
& X_{\Phi}=-\frac{1}{2 \pi\left\langle 0_{j}|\Phi| 0_{j}\right\rangle} \int d^{2} x\left\langle 0_{j}|\Theta(x) \Phi(0)| 0_{j}\right\rangle,
\end{aligned}
$$

allowing the determination of the ultraviolet conformal data (central charge and scaling dimensions) in the form of moments of off-critical correlators.

In Fig. 5 we compare the exact formula for the central charge (1.3) with the result yielded by the two-kink approximation (4.3) in the sum rule (4.4). Notice that $p$ can be considered as a continous parameter in the result of the latter computation, in agreement with the fact that many observables in the theory (1.7) have a continous $p$-dependence. With this remark in mind, in the remaining part of this section we will treat $p$ as a real number $\geq 1$.

For $p=1$, in particular, the two-kink form factor (3.6), (3.13) simply reduces to $2 \pi m^{2}(-1)^{(1+i \theta / \pi) / 2}$. This residual rapidity dependence ensures the normalisation condition (3.14) but is immaterial in the computation of the correlator (4.3). Hence, the theory (1.7) at $p=1$ is free and the two-kink computation for the central charge gives the exact result $C=-2$. Due to the equivalence between the Ising model and a free neutral fermion, the two-kink approximation is exact also for $p=3$ and gives $C=-1 / 2$.

The computation of $X_{\Theta}$ through the sum rule (4.5) requires the knowledge of $\langle\Theta\rangle$. Although this quantity cannot be related to $F_{j, \pm}^{\Theta}(\theta)$ due to the vanishing of the residue (3.4), we dispose of the thermodynamic Bethe ansatz result 25]

$$
\langle\Theta\rangle=-\pi m^{2} \tan \frac{\pi p}{2} .
$$

\footnotetext{
${ }^{2}$ Here and in the following we always refer to connected correlators.

${ }^{3}$ In doing that, of course we loose unitarity unless $p$ is an integer larger than 2 . Here and in the following the term 'unitarity' refers to the absence of negative norm states in the Hilbert space.
} 
Since $\varphi_{1,3}(x)$ is the operator which drives the theory away from criticality, we must have

$$
\Theta(x) \sim \lambda \varphi_{1,3}(x)
$$

and $X_{\Theta}=X_{1,3}$.

The two-kink computation of $X_{\mathcal{E}}$ through (4.5) gives $-1 / 4$ at $p=1$ and $1 / 8$ at $p=3$. Since the theory is free for these two values of $p$, these results are expected to be exact. Assuming that $\mathcal{E}(x)$ corresponds to a primary operator whose position in the Kac's table does not depend on $p$, they can be substituted into the formula (1.4) to fix

$$
\mathcal{E}(x) \sim \varphi_{1,2}(x) .
$$

In Fig. 6 we compare the two-kink approximation for $X_{\Theta}$ and $X_{\mathcal{E}}$ with the exact formulae

$$
\begin{aligned}
& X_{1,3}=2 \frac{p-1}{p+1}, \\
& X_{1,2}=\frac{p-2}{2(p+1)} .
\end{aligned}
$$

respectively. Concerning the two values of $p$ for which we determined the form factors of the order parameter, the two-kink computation gives $X_{\varphi}=1 / 8$ at $p=3$ and $X_{\varphi}=0.0734$ at $p=4$. In view of the identification $\varphi(x) \sim \varphi_{2,2}(x)$, these results must be compared with the exact values $1 / 8$ and $3 / 40=0.075$, respectively.

Some comments are in order about the results yielded by the sum rules (4.4) and (4.5). Consider the moment

$$
I_{k}=\int d^{2} x|x|^{k}\left\langle\Phi_{1}(x) \Phi_{2}(0)\right\rangle,
$$

and denote $\Phi_{3}(x)$ the leading operator determining the short distance behaviour

$$
\left\langle\Phi_{1}(x) \Phi_{2}(0)\right\rangle \sim \frac{\left\langle\Phi_{3}\right\rangle}{|x|^{\eta_{\Phi_{1} \Phi_{2}}}}, \quad|x| \rightarrow 0
$$

with

$$
\eta_{\Phi_{1} \Phi_{2}} \equiv X_{\Phi_{1}}+X_{\Phi_{2}}-X_{\Phi_{3}} .
$$

Then $I_{k}$ is convergent if $2+k-\eta_{\Phi_{1} \Phi_{2}}>0$. In the cases of interest for us we have $\eta_{\Theta \Theta}=2 X_{1,3}$ and $\eta_{\Theta \mathcal{E}}=X_{1,3}$, so that the sum rules for $C$ and $X_{1,2}$ converge for all finite values of $p$, while the sum rule for $X_{1,3}$ converges only for $p<3$. This 'failure' of the sum rule for the scaling dimension due to the divergence of the integral is originated by operator mixing under renormalisation and was discussed in Ref. [27].

Let us now discuss the issue of the accuracy of the results obtained using the approximated correlators (4.3) into the integrals (4.4) and (4.5). The spectral series for 
the correlation functions is a large distance expansion and any partial sum including the contributions up to $n$ particles will appreciably depart from the exact result at sufficiently small distances. In the moment (4.11), however, the factor $|x|^{k}$ causes a suppression of the short distance contribution whose importance, for a fixed $k$, depends on the high energy behaviour of the form factors. The two-kink contribution to $I_{k}$ is given by

$$
\int d \theta \frac{F_{j, \pm}^{\Phi_{1}}(2 \theta) F_{j, \pm}^{\Phi_{2}}(-2 \theta)}{(\cosh \theta)^{k+2}} .
$$

The integrand here behaves asymptotically as $\exp \left[-\Sigma_{\Phi_{1} \Phi_{2}}^{(k)} \theta\right]$, where

$$
\Sigma_{\Phi_{1} \Phi_{2}}^{(k)} \equiv 2+k-y_{\Phi_{1}}-y_{\Phi_{2}}
$$

with $y_{\Phi}$ defined by

$$
F_{j, \pm}^{\Phi}(\theta) \sim e^{y_{\Phi} \theta / 2}, \quad \theta \rightarrow+\infty
$$

In an unitary theory, this exponent is subject to the bound [5]

$$
y_{\Phi} \leq X_{\Phi}
$$

We see that $\Sigma_{\Phi_{1} \Phi_{2}}^{(k)}$ has to be positive to ensure the convergence of the integral and that the suppression of the short distance contribution is proportional to this exponent. Of course, this observation does not determine the absolute accuracy of the two-particle approximation, but it helps understanding the accuracy pattern exhibited in Figs. 6. In fact, the solutions of Section 3 determine

$$
\begin{aligned}
& y_{\Theta}=\frac{3(p-1)}{2 p}, \\
& y_{\mathcal{E}}=\frac{p-3}{2 p} .
\end{aligned}
$$

Then, $\Sigma_{\Theta \Theta}^{(0)}$ goes to zero as $p \rightarrow 3$ and we observe that the deviation of the two-kink approximation for $X_{\Theta}$ from the exact result becomes large as we approach this value. Analogous considerations apply to the case of $X_{\mathcal{E}}$ as $p \rightarrow \infty$. In the central charge sum rule (4.4), on the contrary, $\Sigma_{\Theta \Theta}^{(2)}$ tends to 1 as $p \rightarrow \infty$, what means that the high energy contribution is still strongly suppressed in this limit. The remarkable accuracy (1\%) of the two-kink approximation as $p \rightarrow \infty$ shows that also the contributions with a larger number of kinks undergo a similar suppression. The relation between $\Sigma_{\Phi_{1} \Phi_{2}}^{(k)}$ and the accuracy of the two-kink approximation (defined as the absolute deviation from the exact result divided by the exact result) is illustrated in Table 1 through few examples. 


\section{Double perturbation and phase diagram}

In this section we briefly consider what happens if we add to the action (1.7) the operator $\mathcal{E} \sim \varphi_{1,2}$, namely if we take

$$
\mathcal{A}^{\prime}=\mathcal{A}_{C F T}^{(p)}+\lambda \int d^{2} x \varphi_{1,3}^{(p)}(x)+\mu \int d^{2} x \varphi_{1,2}^{(p)}(x)
$$

Within the usual conventions for the operator normalisations, the regime III we considered in the previous sections corresponds to $\mu=0$ and $\lambda<0$. If $\mu$ is very small we can use form factor perturbation theory [8] around regime III. The correction to the energy density $\varepsilon_{j}$ of the vacuum $\left|0_{j}\right\rangle$ is proportional to the vacuum expectation value of the perturbing operator $\varphi_{1,2}$ computed at $\mu=0$, and reads (remember (3.15) and (3.4))

$$
\delta \varepsilon_{j} \sim \mu\left\langle 0_{j}\left|\varphi_{1,2}\right| 0_{j}\right\rangle=\mu(-1)^{j}\left\langle 0_{1}\left|\varphi_{1,2}\right| 0_{1}\right\rangle
$$

This means that only a subset of alternating vacua among the $p-1$ degenerate vacua of regime III preserves the same energy when $\lambda$ is negative and $\mu$ is small in (5.1). For $p$ odd, in particular, we see that the $Z_{2}$ symmetry characteristic of the case $\mu=0$ is broken by the $\varphi_{1,2}$ perturbation. For $p=3$ the action (5.1) describes the Ising model in a magnetic field [0, 8]. If $p$ is odd the number of surviving degenerate vacua is $(p-1) / 2$. If $p$ is even, instead, this number is $p / 2$ or $p / 2-1$ depending on the sign of $\mu$.

It is clear that in presence of such a pattern of degeneracy breaking the kinks $K_{j, j \pm 1}$ of regime III are no longer asymptotic excitations when $\mu \neq 0$. Rather, they will be confined into pairs $K_{j, j+1} K_{j+1, j+2}$ providing the new stable kinks in the perturbed theory. This phenomenon appears in the formalism when we try to compute the correction to the mass of the kinks $K_{j, j \pm 1}$, which is given by 8,28

$$
\delta m \sim \mu F_{j, \pm}^{\varphi_{1,2}}(i \pi)
$$

Since the form factor on the r.h.s. has a pole at $\theta=i \pi$ (see (3.6) and (3.15)), it follows that this correction is infinite, a fact that reveals the removal of the kinks $K_{j, j \pm 1}$ from the spectrum of the asymptotic excitations.

The conformal field theories with $C<1$ perturbed with one of the operators $\varphi_{1,3}$, $\varphi_{1,2}$ or $\varphi_{2,1}$ are integrable [2]. It is then natural to look for solvable lattice models whose scaling limit corresponds to these quantum field theories. In Ref. 229] a 'dilute' version of the RSOS models was considered and found to be solvable on the lattice along four distinct

\footnotetext{
${ }^{4}$ When useful in this section we explicitely label the operators by the superscript $(p)$ identifying the critical point they refer to.
} 
branches. It was found, in particular, that the scaling limit of 'branch 2' is described by the action (5.1) with $\lambda=0$, and that for $p$ odd this branch possesses $(p-1) / 2$ degenerate ground states. This result is consistent with our perturbative considerations and suggests that they hold true for the whole region $\lambda \leq 0$ in (5.1).

We already mentioned that for $p>3$ the regime IV of the $\operatorname{RSOS}$ models $(\lambda>0$, $\mu=0$ in (5.1)) corresponds to a massless flow to the critical point with action $\mathcal{A}_{C F T}^{(p-1)}$. It is known that the operator $\varphi_{1,2}^{(p)}$ renormalises into the operator $\varphi_{2,1}^{(p-1)}$ in the infrared limit of this flow 21]. Hence we conclude that the action (5.1) in the limit $\lambda=+\infty$ describes the $\varphi_{2,1}^{(p-1)}$ perturbation of the critical point $\mathcal{A}_{C F T}^{(p-1)}$. This integrable perturbation was identified in 29] as corresponding to the scaling limit of the lattice models along 'branch 1泡. The phase diagram associated with the action (5.1) is shown in Fig. 7.

\section{Conclusion}

In the central part of this paper we applied the $S$-matrix-form factor approach to the regime III of the RSOS models. These models, however, are not the only lattice models whose scaling limit is described by the $\varphi_{1,3}$ perturbation of $C<1$ conformal field theories. It is well known [30, 31, 32] that the same action (1.7) corresponds to the scaling dilute $q$ state Potts model at the critical temperature and zero external field (with $q=4 \cos ^{2} \pi / p \in$ $[0,4]$ ), and to the scaling $O(n)$ vector model in zero external field (with $n=2 \cos \pi / p \in$ $[-2,2])$. The latter two models make sense for continous values of $q$ and $n$ through mapping onto cluster and loop models, respectively. Excepting special values of $p(p=3$ in particular) these three models are characterised by different internal symmetries and then represent different universality classes of critical behaviour. In fact, the order parameter has a different number of independent components in the three cases and corresponds to different operators (see Table 2). The fact that the three models are described by the same action along the renormalisation group trajectories specified above means that the theory (1.7) admits different microscopic descriptions distinguished by the choice of local observables?. Each description is characterised by a specific set of mutually local operators with well defined transformation properties under the group of internal symmetry. Of course, the perturbing operator $\varphi_{1,3}$ appears in all these local sets and is invariant under

\footnotetext{
${ }^{5}$ The remaining two solvable branches of Ref. [29] are not related to perturbations of the critical points considered in this paper.

${ }^{6}$ Famous examples of this kind of situation are the equivalence between the Ising model and free neutral fermions, or that between the Sine-Gordon and massive Thirring models.
} 
the different symmetry groups. The observables associated to this operator, e.g. the correlation length critical exponent $\nu=1 /\left(2-X_{1,3}\right)$, are the same in the three cases.

At the conformal level, the possibility of different local descriptions appears through the existence of different modular invariant partition functions for the same value of the central charge $C$ [33, 34, 35]. In the S-matrix approach away from criticality the different nature of the order parameter leads to the existence of different scattering descriptions for the action (1.7). They all exhibit the same spectrum and very similar analytic form but differ from each other for the nature and the number of the elementary excitations (see Table 2). In this paper we used the scattering description based on the $Z_{2}$ symmetry which characterises the RSOS models. The massive dilute $q$-state Potts model at $T=T_{c}$ [12, 36] has $q+1$ degenerate vacua located at the $q$ vertices and at the center of a hypertetrahedron living in the $(q-1)$-dimensional space of the independent order parameter components. The elementary excitations are the $2 q$ kinks interpolating from the center to the vertices, and vice versa (Fig. 8). In the massive phase of the $O(n)$ model there is a single vacuum and the elementary excitations are $n$ ordinary particles transforming according to the vector representation of the group [13, 14]. Of course, for non-integer values of $q$ and $n$ the number of excitations is also non-integer, but this is not more surprising than the appearence of operators with non-integer multiplicity in the modular invariant partition functions for the two models at criticality (see [35]).

The different number of excitations for a given $p$ ensures that there is no one to one correspondence between the three scattering descriptions, although some connections certainly exists 7 . For each particle basis the asymptotic states have obvious transformation properties under the relevant symmetry group and this fact allows a natural identification within the form factor approach of the interesting operators (for example the order parameter).

As was discussed above, no matter which particle basis is used, summation over the intermediate asymptotic states must lead to the same result for the correlation functions of some invariant operators, in particular the trace of the stress-energy tensor $\Theta(x) \sim$ $\lambda \varphi_{1,3}(x)$. Since each $n$-particle contribution to the spectral sum has a distinct large distance behaviour $\exp (-n m r)$, the identification is expected to occur term by term. It is easy to check comparing the results of this paper with those of Refs. 12, 14 that this is indeed the case for the first (two-particle) contribution to $\langle\Theta(x) \Theta(0)\rangle$.

\footnotetext{
${ }^{7}$ A relation between the RSOS and dilute Potts scattering theory for $p=6$ was pointed out in [36]. The issue of the relation between the $O(n)$ and RSOS scattering descriptions has been discussed in [37] from the point of view of quantum group reduction of Sine-Gordon model.
} 
Most of the considerations of this section can be extended to the case of the more general action (5.1).

Acknowledgements. I thank John Cardy for interesting discussions.

\section{References}

[1] A.B. Zamolodchikov, Al.B. Zamolodchikov, Ann.Phys. 120 (1979), 253.

[2] A.B. Zamolodchikov, in Adv. Stud. Pure Math. 19 (1989), 641; Int. J. Mod. Phys. A3 (1988), 743.

[3] M. Karowski, P. Weisz, Nucl. Phys. B 139 (1978), 445.

[4] F.A. Smirnov, Form Factors in Completely Integrable Models of Quantum Field Theory (World Scientific) 1992.

[5] G. Delfino, G. Mussardo, Nucl. Phys. B 455 (1995), 724;

[6] G. Delfino and P. Simonetti, Phys. Lett. B 383 (1996), 327.

[7] G. Delfino, Phys. Lett. B 419 (1998), 291.

[8] G. Delfino, G. Mussardo and P. Simonetti, Nucl. Phys. B 473 (1996), 469.

[9] L. Chim and A.B. Zamolodchikov, Int. J. Mod. Phys. A7 (1992), 5317.

[10] G. Delfino and J.L. Cardy, Nucl. Phys. B 519 (1998), 551.

[11] G. Delfino, G.T. Barkema and J.L. Cardy, cond-mat/9908453, to appear in Nucl. Phys. B.

[12] G. Delfino, Nucl. Phys. B 554 (1999), 537.

[13] A.B. Zamolodchikov, Mod. Phys. Lett. A6 (1991), 1807.

[14] J.L. Cardy and G. Mussardo, Nucl. Phys. B 410 (1993), 451.

[15] G. Delfino, Phys. Lett. B 450 (1999), 196. 
[16] G. Andrews, R. Baxter and J. Forrester, J. Stat. Phys. 35 (1984), 193.

[17] D.A. Huse, Phys. Rev. B 30 (1984), 3908.

[18] A.A. Belavin, A.M. Polyakov and A.B. Zamolodchikov, Nucl. Phys. B 241 (1984), 333.

[19] D. Friedan, Z. Qiu and S. Shenker, Phys. Rev. Lett. 52 (1984), 1575.

[20] A.B. Zamolodchikov, Sov. J. Nucl. Phys. 44 (1986), 529.

[21] A.B. Zamolodchikov, JETP Lett. 43 (1986), 730.

[22] A.W.W. Ludwig and J.L. Cardy, Nucl. Phys. B 285 (1987), 687.

[23] A.B. Zamolodchikov, Landau Institute preprint (1989).

[24] A. LeClair, Phys. Lett. B 230 (1989), 103.

[25] P. Fendley, H. Saleur and Al.B. Zamolodchikov, Int. J. Mod. Phys. A8 (1993), 5751.

[26] J.L. Cardy, Phys. Rev. Lett. 60 (1988), 2709.

[27] G. Delfino, P. Simonetti and J.L. Cardy, Phys. Lett. B 387 (1996), 327.

[28] G. Delfino and G. Mussardo, Nucl. Phys. B 516 (1998), 675.

[29] S.O. Warnaar, B. Nienhuis and K.A. Seaton, Phys. Rev. Lett. 69 (1992), 710.

S.O. Warnaar, P.A. Pearce, K.A. Seaton and B. Nienhuis, J. Stat. Phys. 74 (1994), 469.

[30] Vl.S. Dotsenko and V.A. Fateev, Nucl. Phys B 240 (1984), 312.

[31] B. Nienhuis, J. Phys. A 15 (1982), 199.

[32] B. Nienhuis, Phys. Rev. Lett. 49 (1982), 1062.

[33] J.L. Cardy, Nucl. Phys. B 270 (1986), 186.

[34] A. Cappelli, C. Itzykson and J.B. Zuber, Nucl. Phys. B 280 (1987), 445.

[35] P. Di Francesco, H. Saleur and J.B. Zuber, Nucl. Phys. B 285 (1987), 454. 
[36] A.B. Zamolodchikov, in Fields, Strings and Quantum Gravity, H. Guo, Z. Qiu, H. Tye eds., Gordon \& Breach Science Publishers, 1989, 349.

[37] F.A. Smirnov, Phys. Lett. B 275 (1992), 109. 


\begin{tabular}{|c||c|c|c|}
\hline$\Sigma_{\Phi_{1} \Phi_{2}}^{(k)}$ & 0.5 & 1.5 & 2.5 \\
\hline$\rho_{\Theta \Theta}^{(0)}$ & $\begin{array}{c}0.09 \\
(0.66)\end{array}$ & $\begin{array}{c}0.004 \\
(0.55)\end{array}$ & \\
\hline$\rho_{\Theta \Theta}^{(2)}$ & & $\begin{array}{r}0.005 \\
(0.86)\end{array}$ & $\begin{array}{c}0.0008 \\
(0.66)\end{array}$ \\
\hline$\rho_{\Theta \mathcal{E}}^{(0)}$ & $\begin{array}{c}0.03 \\
(0.86)\end{array}$ & $\begin{array}{c}0.007 \\
(0.66)\end{array}$ & $\begin{array}{c}0.004 \\
(0.55)\end{array}$ \\
\hline
\end{tabular}

Table 1. Accuracy $\rho_{\Phi_{1} \Phi_{2}}^{(k)}$ of the two particle approximation for the $k$-th moment of the correlator $\left\langle\Phi_{1}(x) \Phi_{2}(0)\right\rangle$ for three values of the exponent (4.15). The numbers in parentesis are the corresponding values of $p /(p+1)$.

\begin{tabular}{|c||c|c|c|}
\hline & $\begin{array}{c}\text { RSOS } \\
\text { models }\end{array}$ & $\begin{array}{c}\text { Dilute } \\
\text { Potts model }\end{array}$ & $O(n)$ model \\
\hline & & $q=4 \cos ^{2} \frac{\pi}{p}$ & $n=2 \cos \frac{\pi}{p}$ \\
\hline Symmetry & $Z_{2}$ & $S_{q}$ & $O(n)$ \\
\hline $\begin{array}{c}\text { Order parameter: } \\
\text { number of components } \\
\text { scaling dimension }\end{array}$ & 1 & $q-1$ & $n$ \\
\hline number of vacua & $p-1$ & $q+1$ & 1 \\
\hline number of & $X_{2,2}$ & $X_{\frac{p}{2}, \frac{p}{2}}$ & $X_{\frac{p-1}{2}, \frac{p+1}{2}}$ \\
elementary excitations & $2(p-2)$ & $2 q$ & $n$ \\
\hline
\end{tabular}

Table 2. Some features of the lattice models whose continuum limit is described by the action (1.7). The notation for the scaling dimensions refers to (1.4). 


$\begin{array}{llllllllllll}1 & 2 & 1 & 2 & 3 & 2 & 3 & 2 & 3 & 4 & 3 & 4 \\ 2 & 1 & 2 & 1 & 2 & 3 & 2 & 3 & 4 & 3 & 4 & 3 \\ 1 & 2 & 1 & 2 & 3 & 2 & 3 & 2 & 3 & 4 & 3 & 4 \\ 2 & 1 & 2 & 1 & 2 & 3 & 2 & 3 & 4 & 3 & 4 & 3\end{array}$

Figure 1. The ground states 1,2 and 3 of regime III for $p=4$.

\begin{tabular}{llll|llll}
1 & 2 & 1 & 2 & 3 & 2 & 3 & 2 \\
2 & 1 & 2 & 1 & 2 & 3 & 2 & 3 \\
1 & 2 & 1 & 2 & 3 & 2 & 3 & 2 \\
2 & 1 & 2 & 1 & 2 & 3 & 2 & 3
\end{tabular}

Figure 2. Domain wall (segment) between the ground states 1 and 2. It corresponds to the space-time trajectory of the kink $K_{12}$. 

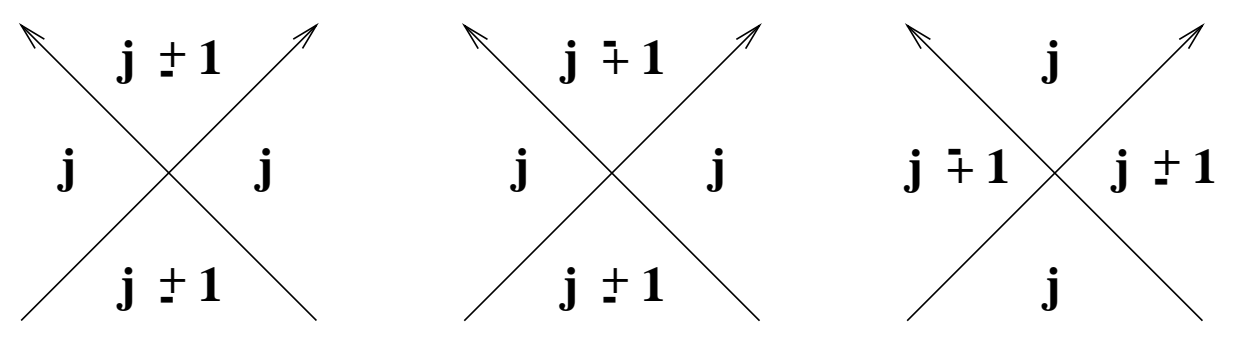

Figure 3. The two-kink scattering amplitudes $A_{j}^{ \pm}, B_{j}$ and $C_{j}$.

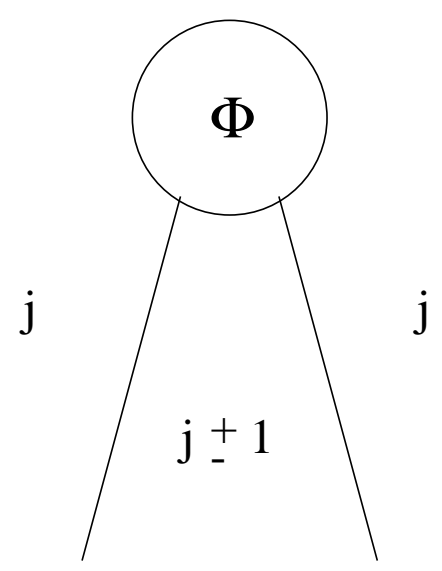

Figure 4. The two-kink form factor $F_{j, \pm}^{\Phi}$ of the operator $\Phi$. 


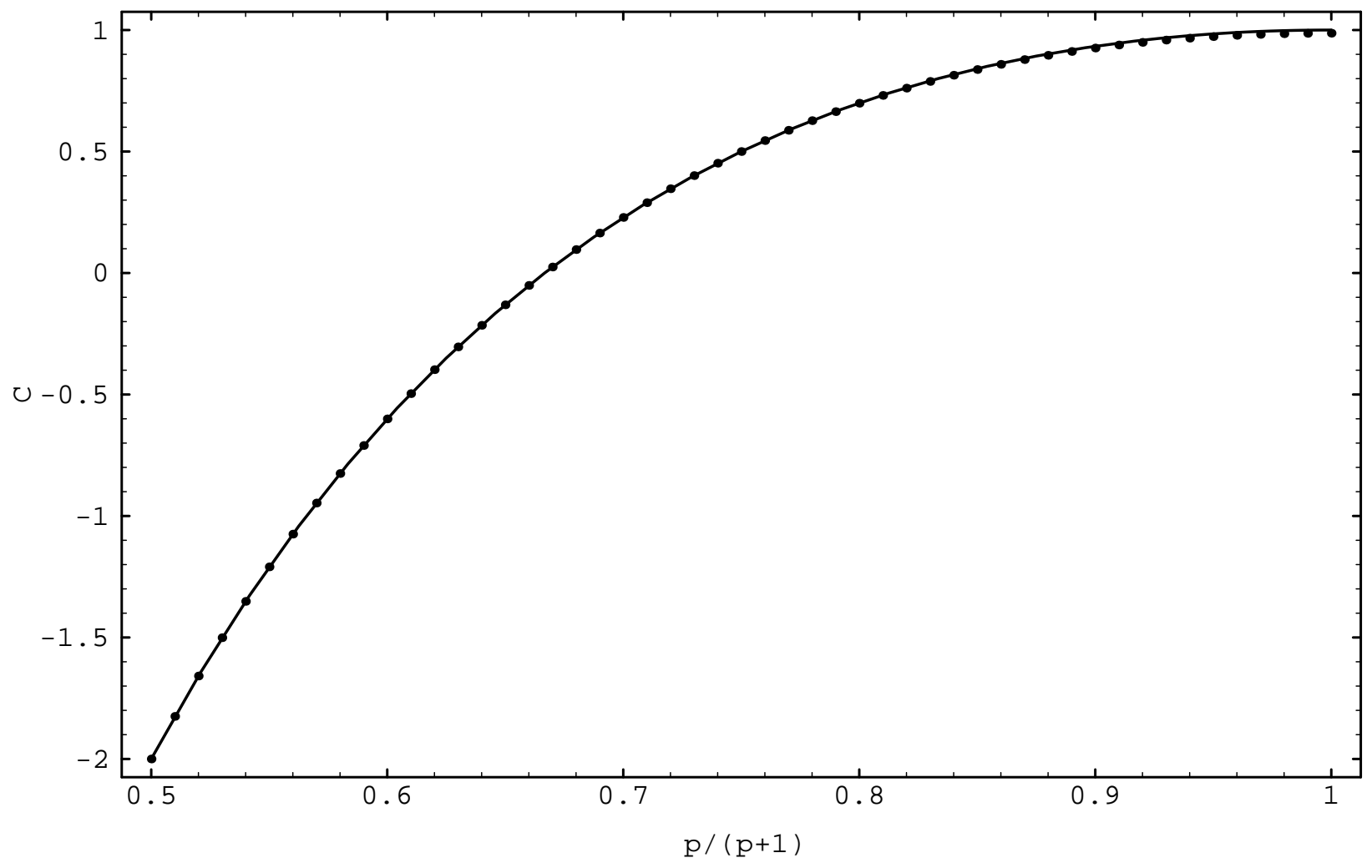

Figure 5. The two-kink approximation for the central charge sum rule (4.4) (dots) compared with the exact formula (1.3) (continous line). 


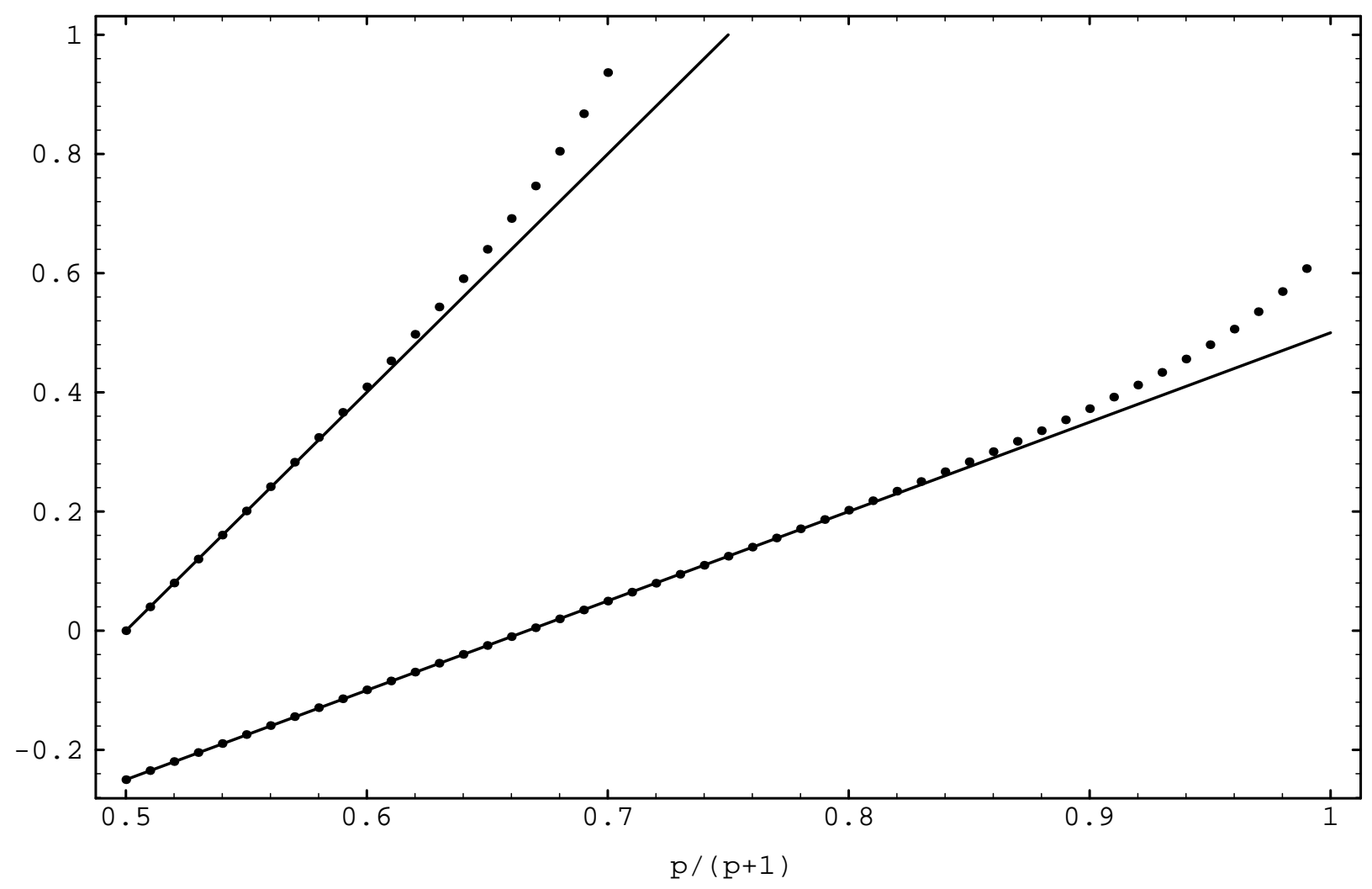

Figure 6. The two-kink approximations for the scaling dimension sum rule (4.5) for the operators $\Theta(x)$ (upper dotted line) and $\mathcal{E}(x)$ (lower dotted line). They are compared with the exact formulae (4.9) and (4.10), respectively (continous lines). 


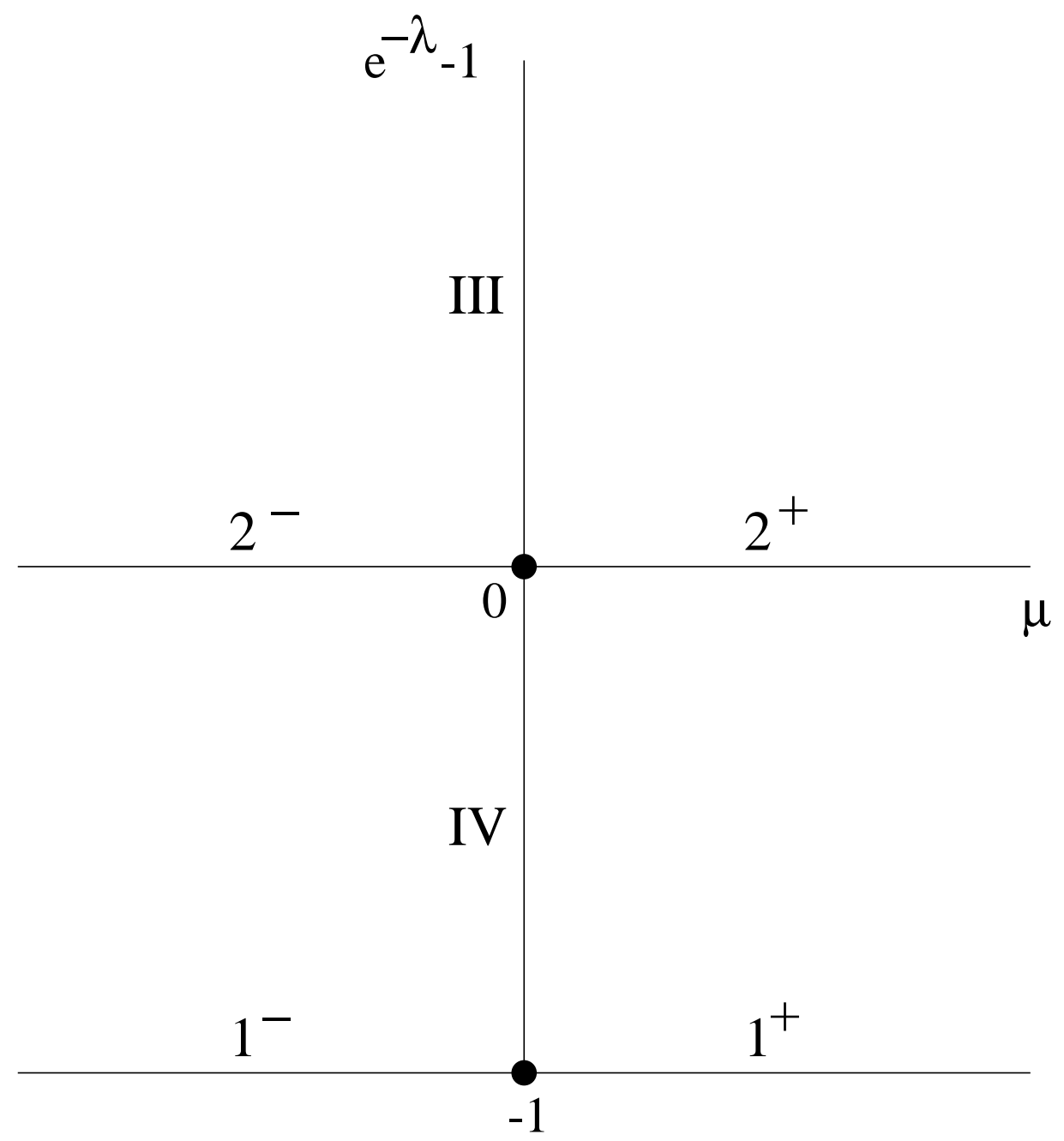

Figure 7. Phase diagram associated to the action (5.1) and illustrating the different integrable branches of the RSOS models.
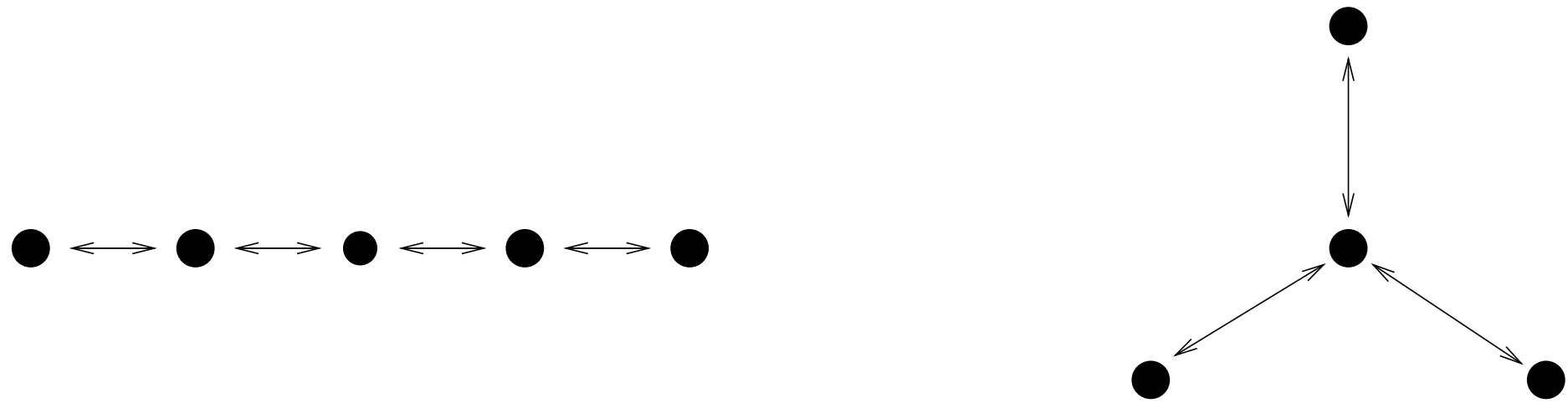

(a)

(b)

Figure 8. Vacua and kinks for $p=6$ in the regime III of the RSOS models (a), and in the massive dilute Potts model at $T=T_{c}(\mathrm{~b})$. 